\title{
A REGIONAL AUDIT OF SYPHILIS
}

1 - Arrowside Unit, Department of Integrated Sexual health, Worcestershire Health and Care Trust

2 - Cobridge Sexual Health Centre, Staffordshire and Stoke on Trent Partnership NHS Trust

On behalf of the West Midlands regional audit group

\section{Background}

BASHH guidelines for syphilis management were revised in 2015 and have recently been published.

\section{Aims/ objectives}

Once the guidelines were published for

consultation, the regional audit committee decided to review regional clinics' syphilis management and adherence to provisional audit standards.

The audit standards are:

- A record of a RPR or VDRL titre obtained pretreatment (standard 97\% confirmed syphilis cases).

- The percentage of confirmed syphilis cases having fully adhered to a recommended treatment (standard 97\% confirmed syphilis cases).

- The percentage of confirmed syphilis cases having a record of a RPR or VDRL titre obtained six months post-treatment (standard 65\% confirmed syphilis cases).

- The ratio of contacts per index case of confirmed syphilis, whose attendence is verified by HACW.

Furthermore it was decided to include a number of other standards of care as part of the audit these included:

- Management of syphilis in pregnancy

- Testing for other STIs

- DGM performed?

- Clinical and chronological stage documented

- First line treatment given

- Jarisch Herxheimer $(\mathrm{JH})$ reaction documentation

\section{Methods}

Regional sexual health clinics were asked to review cases of syphilis diagnosed the previous year with respect to gender, sexuality, HIV status, pregnancy, screening for other sexually transmitted infections, disease stage, whether non treponemal titres were measured, follow up, treatments given, discussion of the Jarisch Herxheimer $(\mathrm{JH})$ reaction and partner notification (PN).

Clinics were asked to complete survey monkey questionnaire in all case of syphilis diagnosed from September 2013-September 2014.

\section{Discussion/ Conclusion}

Although most of the audit standards were reached, there remains area for improvement - these include; demonstration of success of treatment; a discussion of the $\mathrm{JH}$ reaction; a decrease in patients lost to follow up; encouragement of routine communication with obstetric and neonatology teams for all pregnant patients with a current or prior history of syphilis'. A reaudit is planned in the future.

\section{Results}

13/15 (86\%) clinics participated. 161 case notes were reviewed. $81 \%$ were male, 54\% were classified as men having sex with men. 34/161 (21\%) were HIV positive (Fig 1 and Fig 2). 13/161 (19\%) were pregnant.

Fig 1 - Patient sexuality

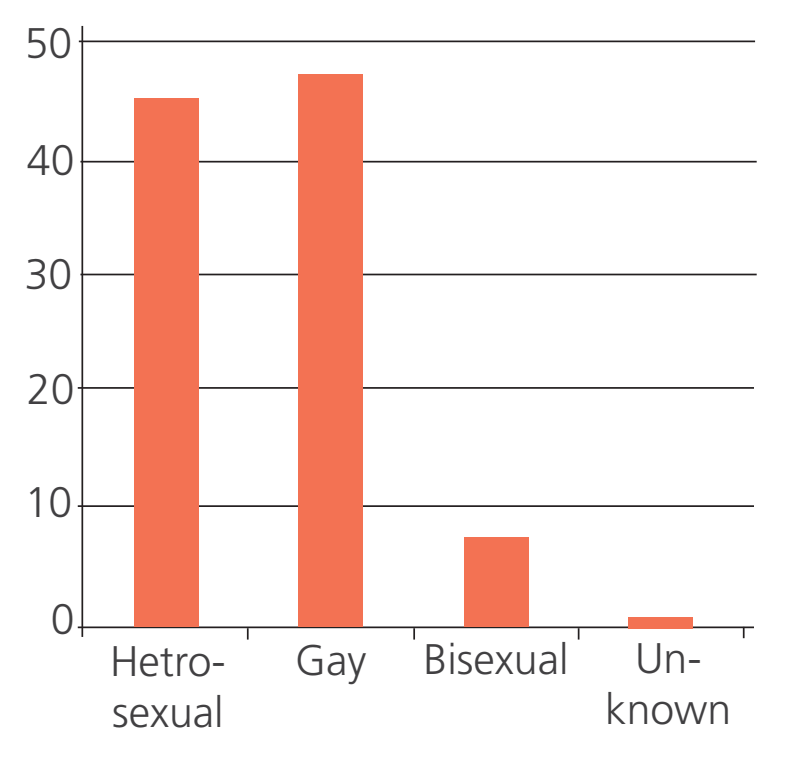

Was the patient screened for other STIs including HIV ?

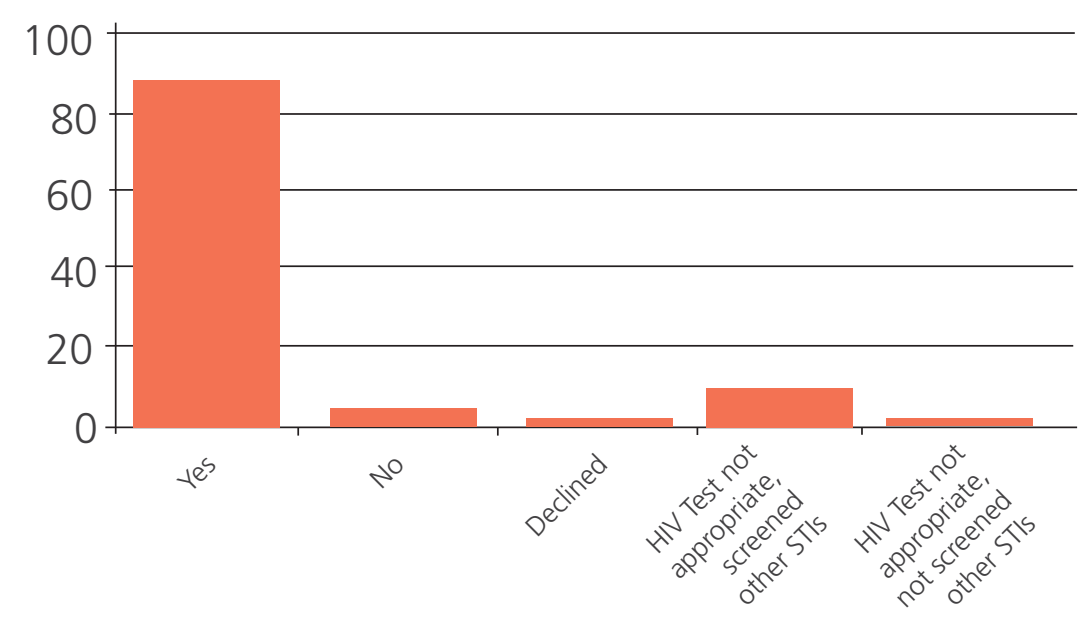

Fig 2 - Patient HIV status

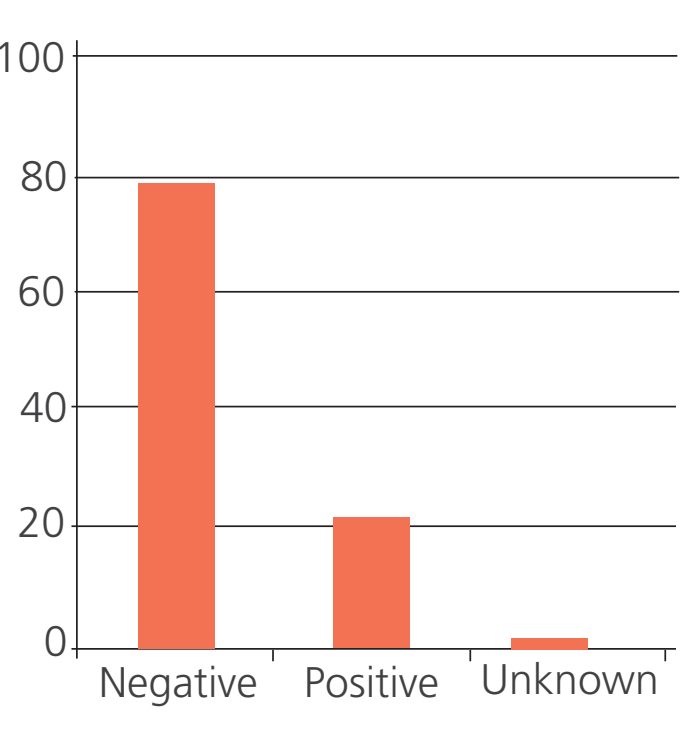

Was communication made with the obstetrics team? Pregnant patients

13/161 (19\%) were pregnant (in $84 \%$ written communication had been made to obstetric/ neonatal teams).

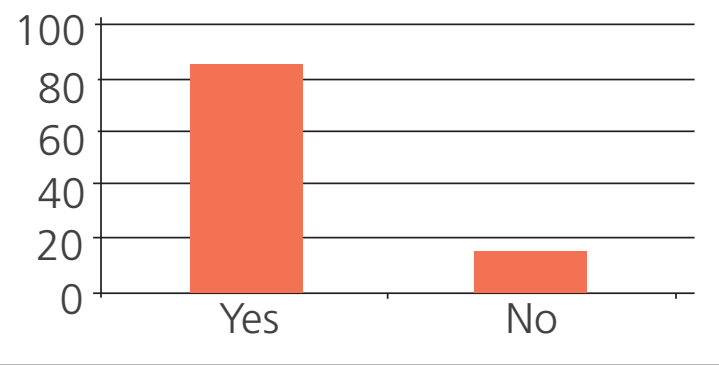

$\begin{array}{ll}\text { Were } & \text { These include } 13 \text { cases of } \\ \text { there any } & \text { chlamydia including rectal }\end{array}$ concomitant chlamydia and -1 case of STIs? lymphogranulum venereum $801-11$ cases of gonorrhoea - 11 cases of HIV (although the data couldn't distinguish cases already diagnosed and new cases

- 6 cases of genital herpes

- 1 case of Trichomonas Vaginalis (TV) , 1 contact of TV

Was dark ground microscopy What was the clinical performed?

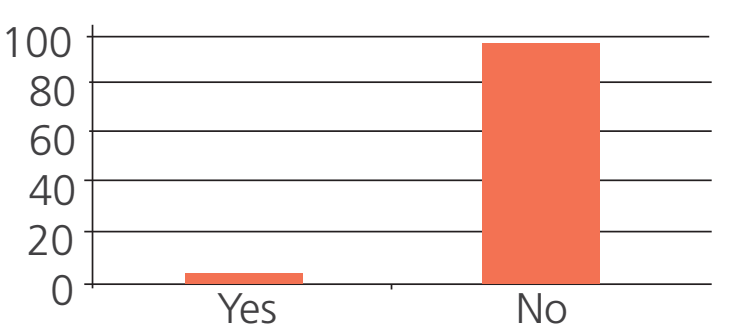

Was the patient treated with parenteral penicillin as per BASHH guidance? $(95 \%$ should complete treatment for early syphilis)?

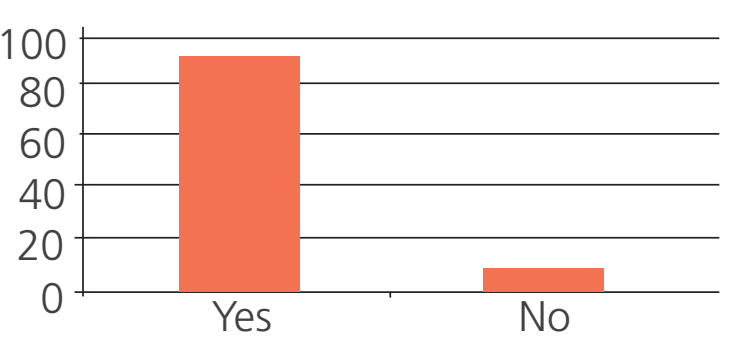

If treated for early syphilis was there documented discusion around the $\mathrm{JH}$ reaction?

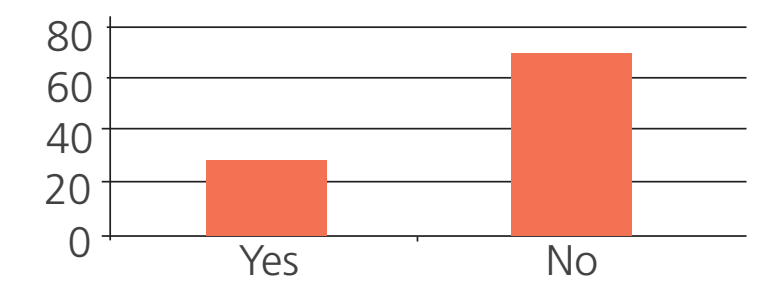
presentation?

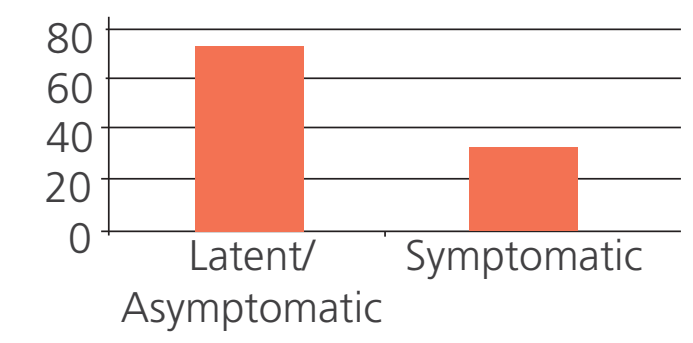

Was neurosyphilis diagnosed?

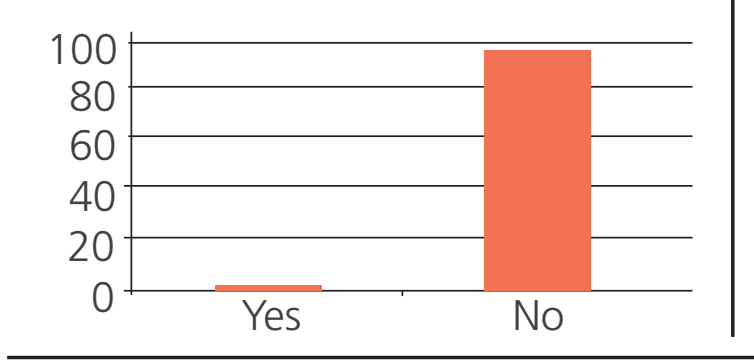

Was there a satisfactory treatment response (ie 4 fold reduction in early syphilis or serofast in late latent syphilis)?

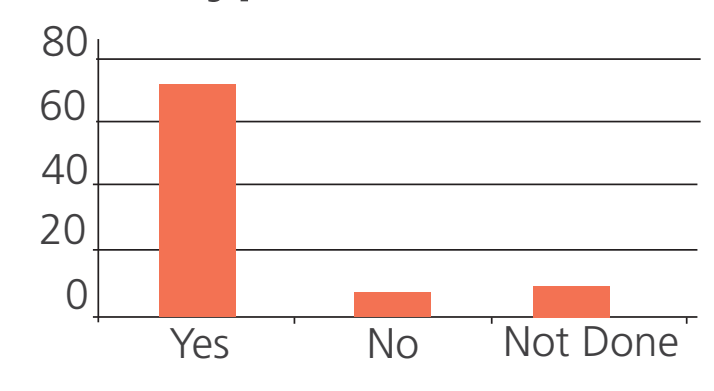

Was partner notification performed?

Partner notification

0.54 of contacts verified as

screened and treated (standard 0.4)
What was the Syphilis chronological stage?

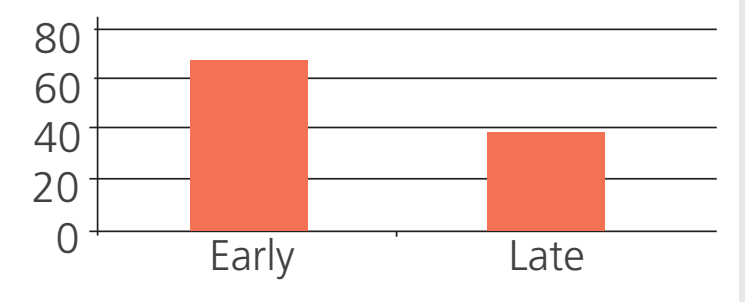

Was an RPR/VDRL performed at commencement of therapy ?(standard 97\%)

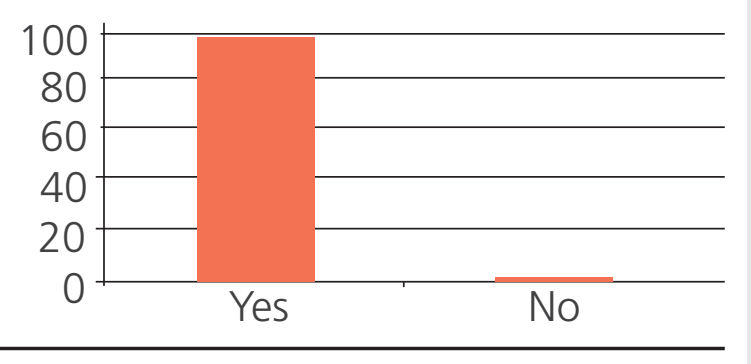

Was an RPR/

VDRL performed at commencement of therapy? (standard 97\%) Follow up post treatment

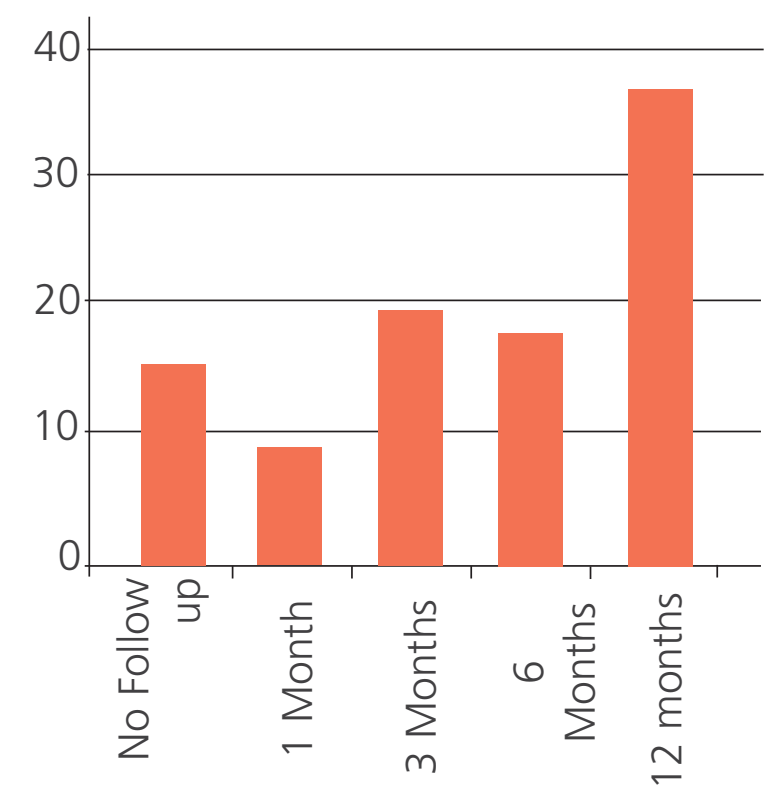

We would like to thank Birmingham Umbrella sexual health service (previous Whittal St and Heartlands), Delia Morris Centre, Burton, Stratford and Warwick sexual health centres (previous Jephson centre), Shrewsbury and Telford sexual health centres, Walsall sexual health centre, The John Anthony Centre, Sandwell sexual health centre, Integrated sexual health services, Hereford, The Arrowside Unit, Integrated sexual health services Coventry and Cobridge sexual health centre, Stafford and Stoke Partnership Trust. 